NASA Technical Memorandum 105395

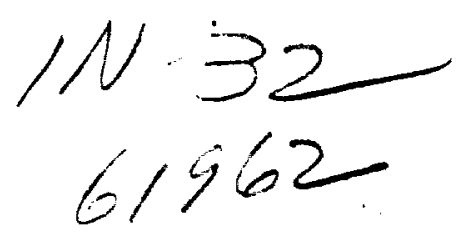

AIAA-92-2018

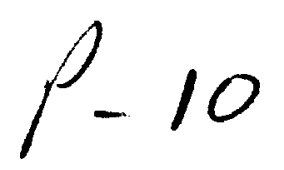

\title{
Interference Susceptibility Measurements for an MSK Satellite Communication Link
}

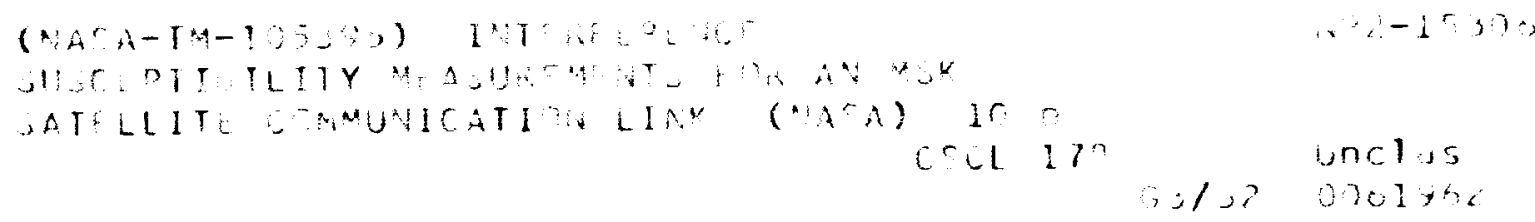

Robert J. Kerczewski and Gene Fujikawa

Lewis Research Center

Cleveland, Ohio

Prepared for the

14th International Communications Satellite Systems Conference sponsored by the American Institute of Aeronautics and Astronautics Washington, D.C., March 22-26, 1992 


\title{
INTERFERENCE SUSCEPTIBILITY MEASUREMENTS FOR
}

\section{AN MSK SATELLITE COMMUNICATION LINK}

\author{
Robert J. Kerczewski and Gene Fujikawa \\ National Aeronautics and Space Administration \\ Lewis Research Center \\ Cleveland, Ohio 44135
}

\begin{abstract}
$\underline{\text { Abstract }}$
This paper presents the results of measurements of the degradation of an MSK satellite link due to modulated and $\mathrm{CW}$ (unmodulated) interference. These measurements were made using a hardware based satellite communication link simulator at NASA Lewis Research Center. The results indicate the amount of bit error rate degradation caused by $\mathrm{CW}$ interference as a function of frequency and power level, and the degradation caused by adjacent channel and cochannel modulated interference as a function of interferer power level. Results were obtained for both the uplink case (including satellite nonlinearity) and the downlink case (linear channel).
\end{abstract}

\section{$\underline{\text { Introduction }}$}

Satellite communication links are subject to interference from various space-based and terrestrial sources. These sources create interference at various relative power levels both within the channel of the desired signal and in adjacent channels.

The SITE Space Communication System Simulator has been developed at NASA's Lewis Research Center to enable the evaluation and verification of technologies related to high frequency (Ka Band), high rate (>200 Mbps) space communication links and networks. ${ }^{1,2}$ The simulator allows the evaluation of components, subsystems, and networks, primarily in terms of the end-to-end system bit-error rate (BER). In particular, the SITE system has the ability to simulate various interference environments and quantify the effects of the interference on system performance.
In order to develop a set of measured data on the performance degradation due to interference for a minimum shift keyed (MSK) satellite link, a series of measurements were performed using the SITE simulator. One set of measurements consisted of cochannel and adjacent channel MSK-modulated interference at several power levels. A second set of measurements consisted of continuous wave (CW), i.e., unmodulated interference, at several power levels and several frequencies relative to the frequency of the desired signal. For both cases, measurements were made to simulate uplink and downlink interference separately. Noise was added to allow BER versus energy-per-bit to noise power density ratio $\left(\mathrm{E}_{\mathrm{b}} / \mathrm{N}_{\mathrm{o}}\right)$ curves to be measured. The results of these measurements are presented below, following a description of the measurement system.

\section{Description of Measurement System}

The MSK modulator and demodulator used for these measurements are described in Figs. 1 and 2. In order to ease implementation at the required data rate of $220 \mathrm{Mbps}$, the modulator and demodulator were designed in a serial, rather than the more familiar quadrature fashion, as described in Ref. 3, leading to the designation serial MSK (SMSK). These modems have been combined with ground terminal control circuitry to form a complete digital ground terminal, operating at $220 \mathrm{Mbps}$. The ground terminal also contains a noise insertion and $E_{b} / N_{a}$ measurement subsystem. Finally, the ground terminal can create a pseudorandom data pattern for modulation and transmission and count errors in the received demodulated data stream. ${ }^{4}$ This BER measurement is accomplished under computer control, with the result being a 
complete BER versus $E_{b} / N_{0}$ curve measured from about $10^{-2}$ to $10^{-8} \mathrm{BER}^{5}$

A simplified block diagram of the SITE simulator is shown in Fig. 3. For the purpose of describing the interference measurements, it suffices to show the satellite transponder as consisting of primarily the TWT, operated at saturation. The TWT represents the only limiter in the transponder. As a result, the uplink interference measurements include the effects of transponder nonlinearity, while the downlink interference measurements do not. No channel filtering was used on the transponder; the available bandwidth was $2.5 \mathrm{GHz}$. Similarly, no channel filters were placed in the ground terminal downconverter. All channel filtering was accomplished in the demodulator. A complete characterization of the channel has been performed. ${ }^{6}$ The transponder operating conditions and frequencies were chosen for optimum system performance; the BER curve measured with no interference present fell within $1 \mathrm{~dB}$ of the theoretical curve (at a BER of $10^{-6}$ ).

The data source, modulator, demodulator, and BER measurement system reside in the digital ground terminal as described above. The interfering signals were combined with the "desired" signal as shown in Fig. 3, and the relative power levels of the interferers are measured at these points.

The CW interferers were placed at 17 frequencies relative to the desired signal, as illustrated in Fig. 4. The main lobe of the SMSK spectrum has a width of 1.5 times the data rate (in this case, $330 \mathrm{MHz}$ ). Note that MSK is in the class of continuous phase frequency shift keyed modulation, with the signaling frequencies at \pm 0.25 times the data rate. At $220 \mathrm{Mbps}$, this corresponds to $\pm 55 \mathrm{MHz}$ from the center of the spectrum. Interferer spacing was thus chosen at $55 \mathrm{MHz}$ so these frequencies would be interfered with directly. In the coherent, serially implemented scheme used for these measurements, the lower signal $(-55 \mathrm{MHz})$ is the carrier. The adjacent channel modulated interferers were centered at $\pm 330 \mathrm{MHz}$ from the center of the desired signal. This is equivalent to having no guard band between the main spectral lobes of the two adjacent signals.

\section{Results for CW Interference}

The downlink $\mathrm{CW}$ interference results are presented in Fig. 5. Although measurements were made for interferers at frequencies from -440 to $+440 \mathrm{MHz}$ relative to the center of the desired signal, no degradation was measured below $-220 \mathrm{MHz}$ or above $+220 \mathrm{MHz}$ at any power level, and therefore these data are not shown.

The four curves in Fig. 5 represent the BER degradation (compared to the case where no interferer was present, at $\mathrm{BER}=10^{-6}$ ) as a function of interferer frequency, relative to the center frequency of the desired modulated signal, for four interferer relative power levels. The results indicate that downlink interference levels below $-25 \mathrm{dBc}$ produce negligible degradation of the BER. At -15 dBc, the CW interference produces degradation of about $2 \mathrm{~dB}$ within the center one-third of the SMSK spectrum (main spectral lobe). Levels above $-15 \mathrm{dBc}$ produce significant degradation across the entire SMSK spectrum, with interferers above $-10 \mathrm{~dB}$ causing complete loss of data.

The results for the uplink $\mathrm{CW}$ interference are presented in Fig. 6. The four curves represent the BER degradation as a function of frequency for four interferer relative power levels. For uplink interference, the relative signal must be $-30 \mathrm{dBc}$ or lower to have negligible effect. Levels of $-20 \mathrm{dBc}$ and above cause severe degradation or complete loss of data.

The uplink interference has significantly greater effect on the system BER than the downlink interference for a given relative power level. This is due to the nonlinear satellite transponder which is present in the uplink measurement but not in the downlink measurement. The nonlinearity contributes intermodulation distortion to the interference 
effects, so that even interferers well outside of the channel (in the absence of channel filtering) can affect the system performance.

\section{Results for Modulated Interference}

Modulated interference measurements were made for an adjacent channel both above and below the desired channel. As Fig. 4 shows, the SMSK spectrum is somewhat asymmetrical, with the first side lobe above the main lobe about $5 \mathrm{~dB}$ higher than the first side lobe below the main lobe, due to the serial method of implementing the MSK modulation. Thus, slightly more degradation is experienced for the adjacent channel interferer below (for the uplink case with the nonlinear transponder present).

The uplink adjacent channel results are plotted in Figs. 7 and 8, with the data presented as BER versus $E_{b} / N_{0}$ curves for each relative power level tested. The adjacent channel interferer below caused $0.5 \mathrm{~dB}$ greater degradation than the interferer above the desired signal at the highest power level tested $(-5 \mathrm{dBc})$. For the adjacent channel below, interference below a level of $-15 \mathrm{dBc}$ caused no measurable degradation. The adjacent channel below caused $1.3 \mathrm{~dB}$ of degradation at a level of $-5 \mathrm{dBc}$. For the adjacent channel above the desired signal, levels below $-5 \mathrm{dBc}$ caused less than $0.4 \mathrm{~dB}$ degradation. At $-5 \mathrm{dBc}$, the degradation was $0.8 \mathrm{~dB}$. These relatively small amounts of degradation would likely be reduced by effective channel filtering at the transponder input.

The uplink cochannel interference results are presented in Fig. 9, for five different power levels. The results show that a cochannel interferer as low as $-30 \mathrm{dBc}$ causes $0.8 \mathrm{~dB}$ of degradation. The degradation increases to $1.7 \mathrm{~dB}$ at $-20 \mathrm{dBc}$ and $3.3 \mathrm{~dB}$ at $-15 \mathrm{dBc}$. Above $-15 \mathrm{dBc}$, the effects of the cochannel interference are severe.

The downlink adjacent channel results are shown in Figs. 10 and 11, for four power levels. For levels as high $0 \mathrm{dBc}$, the degradation is less than $0.6 \mathrm{~dB}$. The results are similar for the adjacent channel both below and above the desired signal. In the absence of the nonlinearity, the demodulator can effectively filter out the adjacent channel interference.

The downlink cochannel interference results are plotted in Fig. 12 for three power levels. At $-30 \mathrm{dBc}$, the degradation is negligible. At $-20 \mathrm{dBc}$ the cochannel interferer causes about $0.8 \mathrm{~dB}$ of degradation, and at $-15 \mathrm{dBc}$ the degradation is $1.5 \mathrm{~dB}$. Degradation becomes severe above $-10 \mathrm{dBc}$. Compared to the uplink case, the downlink channel is about $10 \mathrm{~dB}$ more tolerant of cochannel interference, due to the added intermodulation distortion created by the nonlinear transponder for the uplink case.

\section{Conclusions}

The results of interference degradation measurements made at NASA Lewis Research Center have been presented. These measurements were made on a hardware-based satellite link simulator using $220 \mathrm{Mbps}$ SMSK modulation. In general, the results indicate that the downlink is significantly less susceptible to interference than the uplink. This is due to the nonlinearity existing in the satellite transponder, which is present in the uplink interference measurements but not the downlink. Effects of $\mathrm{CW}$ interference are negligible below $-30 \mathrm{dBc}$ on the uplink, and are negligible below $-20 \mathrm{dBc}$ on the downlink. Adjacent channel modulated interference effects are negligible for the downlink, and relatively small on the uplink. Susceptibility to cochannel interference is also about $10 \mathrm{~dB}$ less on the downlink than on the uplink. Overall, these measurements indicate that the MSK satellite channel can handle reasonably high levels of out-of-band interference, with some improvement likely when channel filtering is added to the transponder. For the cochannel case, levels of $-30 \mathrm{dBc}$ and above on the uplink and $-20 \mathrm{dBC}$ and above on the downlink cause measurable degradation. 


\section{$\underline{\text { References }}$}

1. Kerczewski, R.J., Ivancic, W.I., and Zuzek, J.E., "Evaluation of Components, Subsystems, and Networks for High Rate, High Frequency Space Communications," AlAA Paper 91-3423, Sept. 1991.

2. Ivancic, W.D., Andro, M., Nagy, L.A., Budinger, J.M., and Shalkhauser, M.J., "Satellite-Matrix-Switched, TimeDivision-Multiple-Access Network Simulator," NASA TP-2944, 1989. Presented at the 13th AIAA International Communication Satellite System Conference, Mar. 11-15, 1990.

3. Ziemer, R.E., and Ryan, C.R., "Minimum Shift Keyed Modem Implementation for High Data Rates," IEEE Communications Magazine, Vol. 21, No. 7, Oct. 1983, pp. 28-37.

4. Shalkhauser, M.J., and Budinger, J.M., "Digitally Modulated Bit-Error Rate
Measurement System for Microwave Component Evaluation," NASA TP-2912, 1989.

5. Kerczewski, R.J., Daugherty, E.S., and Kramarchuk, I., "Automated Measurement of the Bit-Error Rate as a Function of Signal-to-Noise Ratio for Microwave Communication Systems," NASA TM-89898, 1987. Presented at the 29th Automatic RF Techniques Group Conference, June 1987.

6. Kerczewski, R.J., and Fujikawa, G., "Performance Measurements for a Laboratory-Simulated $30 / 20 \mathrm{GHz}$ Communication Satellite Transponder," AIAA International Communication Satellite Systems Conference and Exhibit, 13th, AIAA; Part 1, 1990, pp. 277-284. (See also, NASA TM-102424, 1990.)

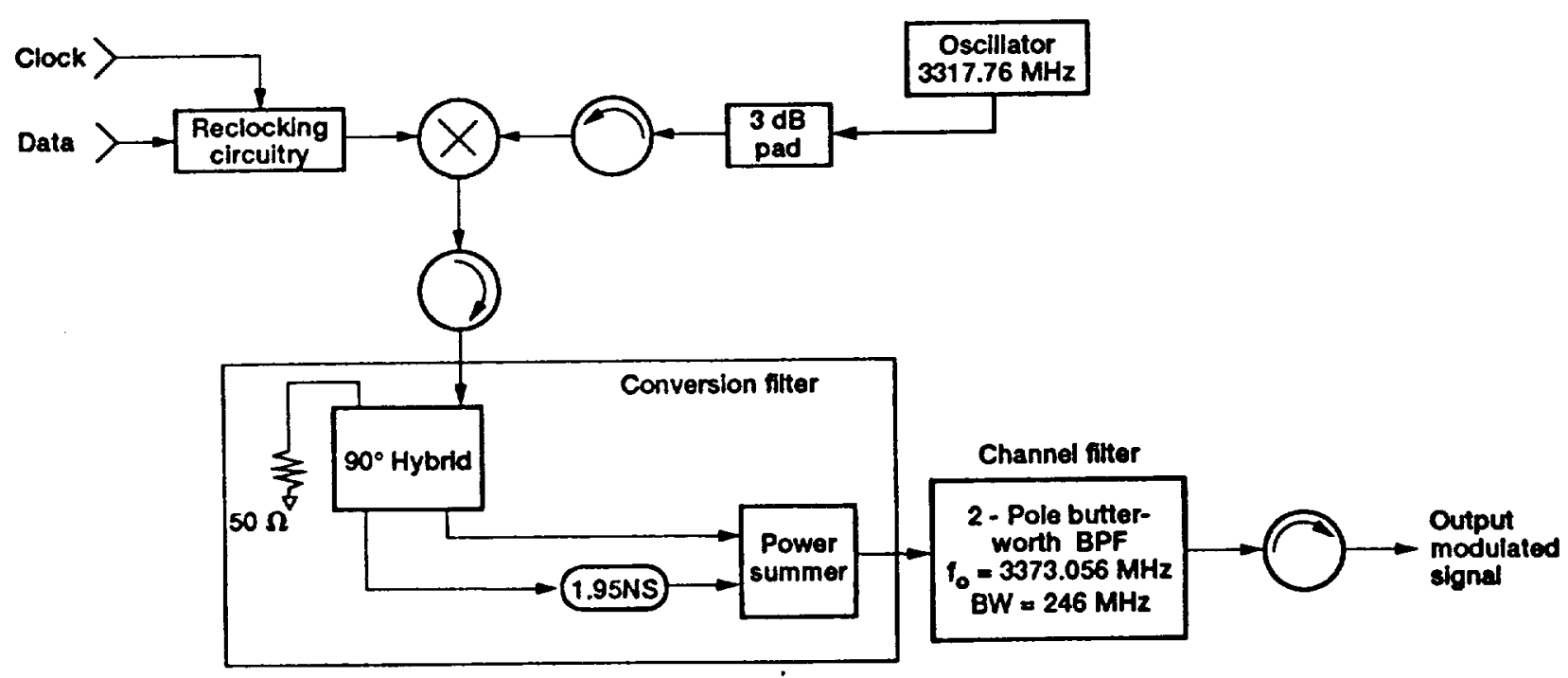

Figure 1.-SMSK modulator block dlagram. 

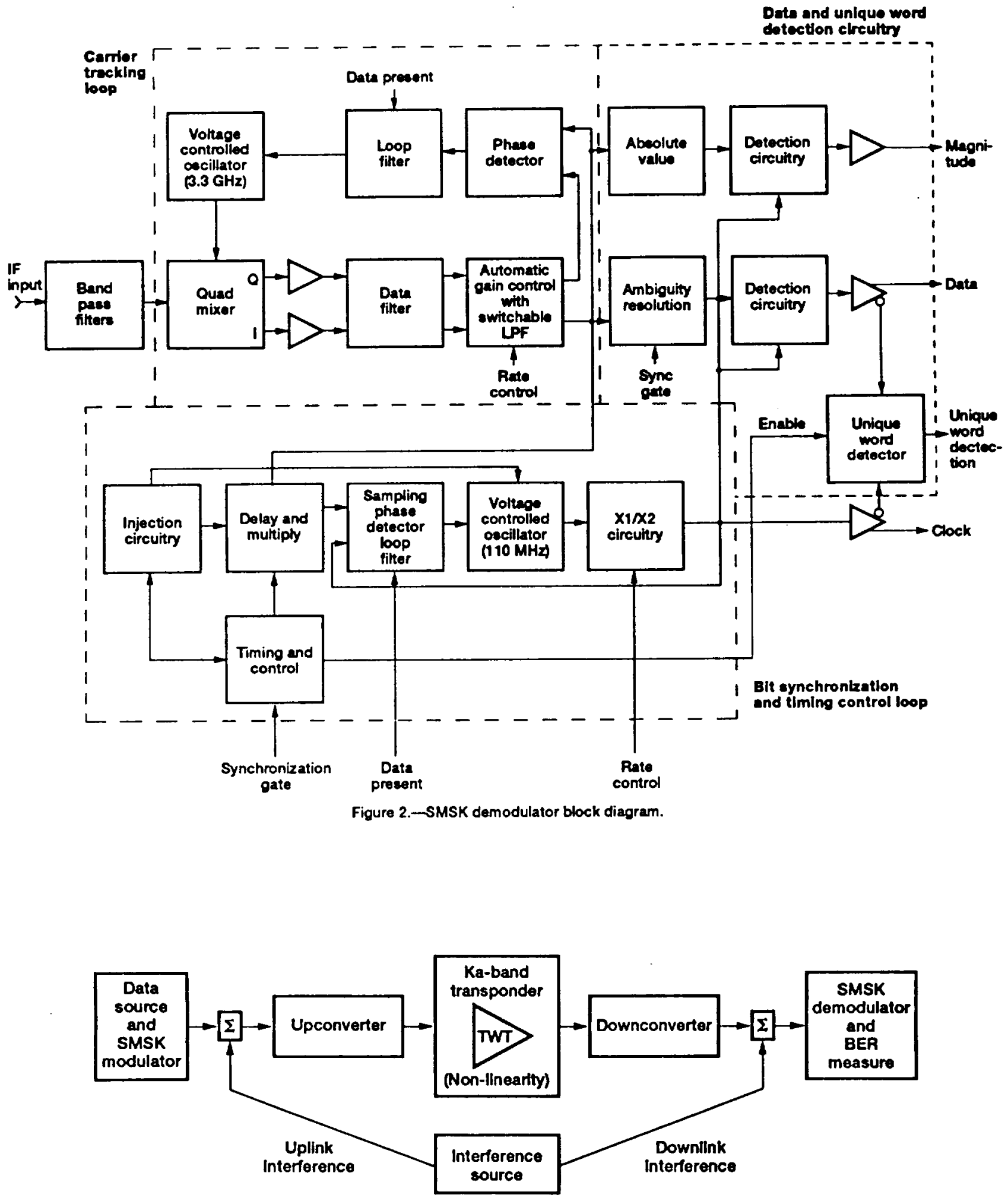

Figure 3.-Interference measurement setup. 


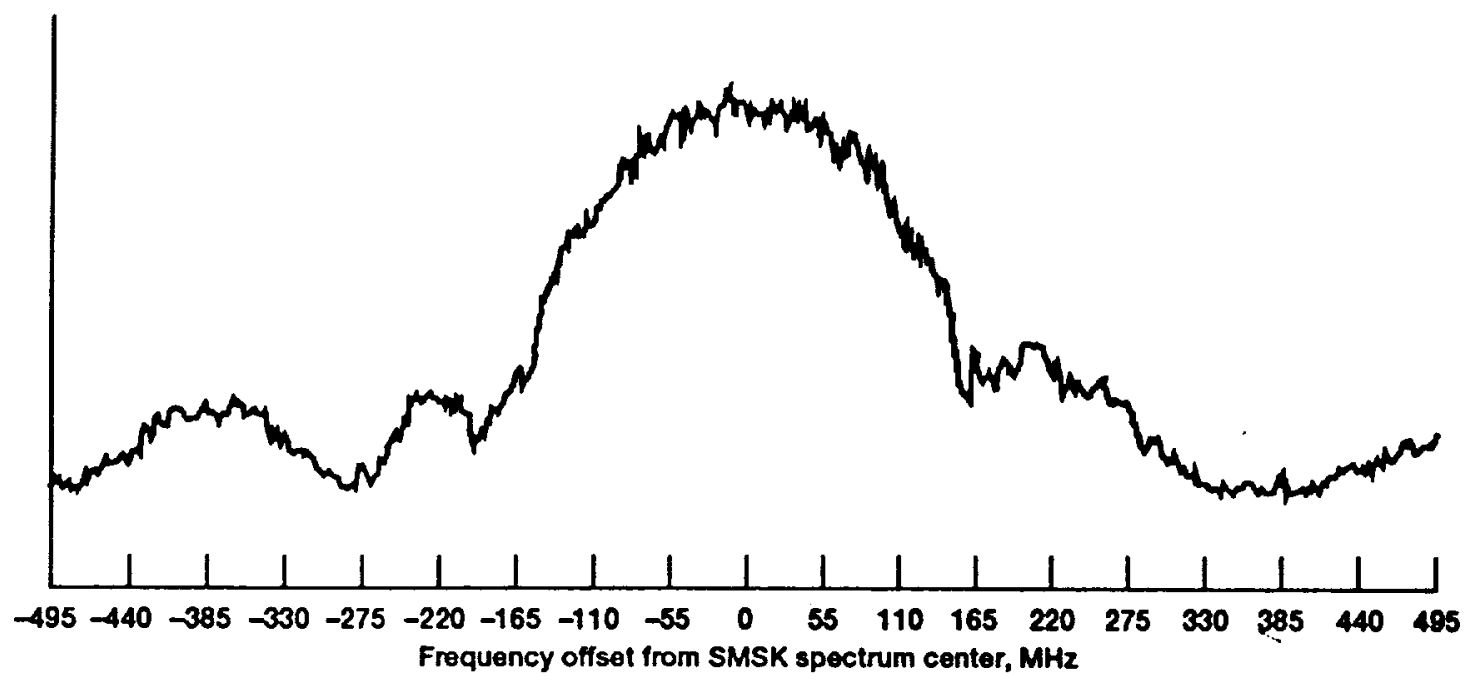

Figure 4.-SMSK modulated spectrum and spectral location of interferers.

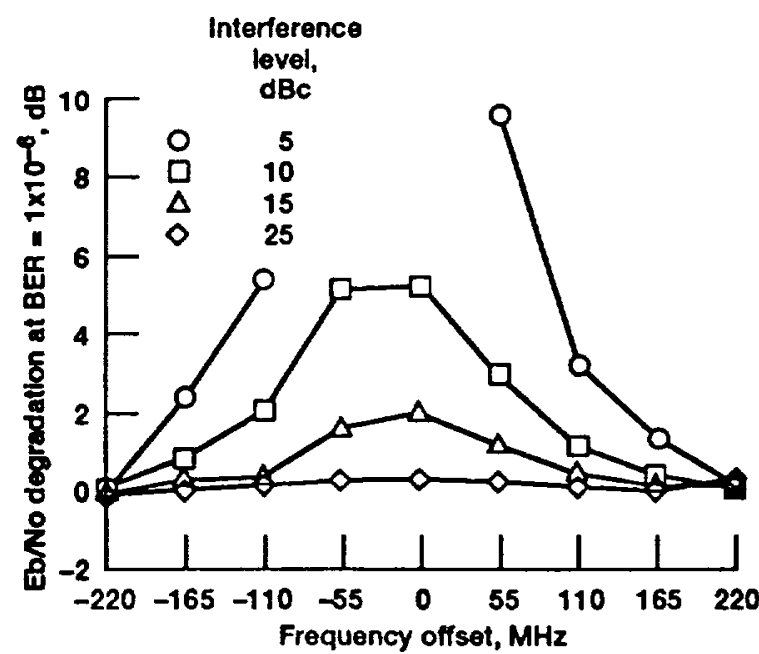

Figure 5.-BER degradation for CW downlink interference as a function of interferer frequency and relative power level.

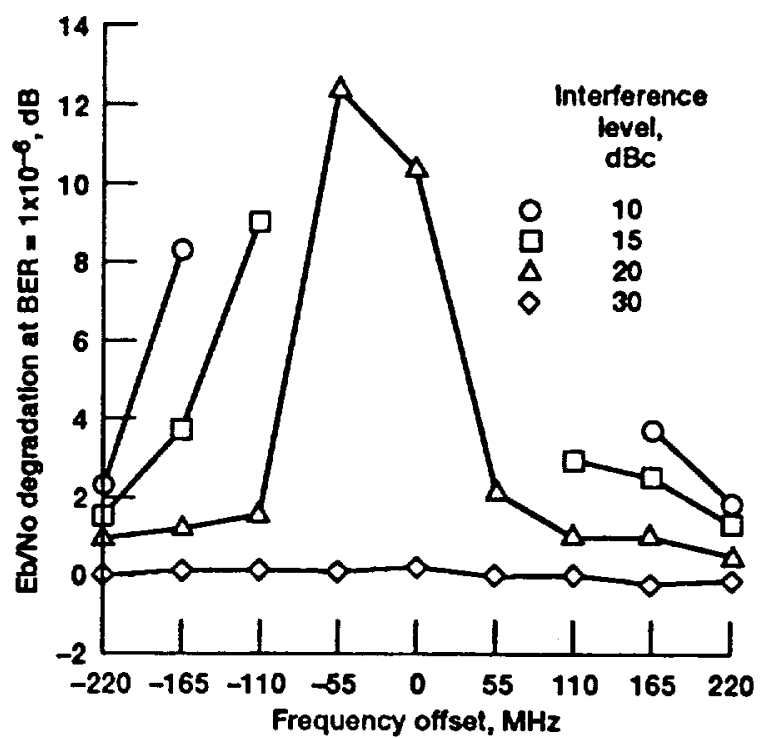

Figure 6.-BER degradation for CW uplink Interference as a function of interferer frequency and relative power level. 


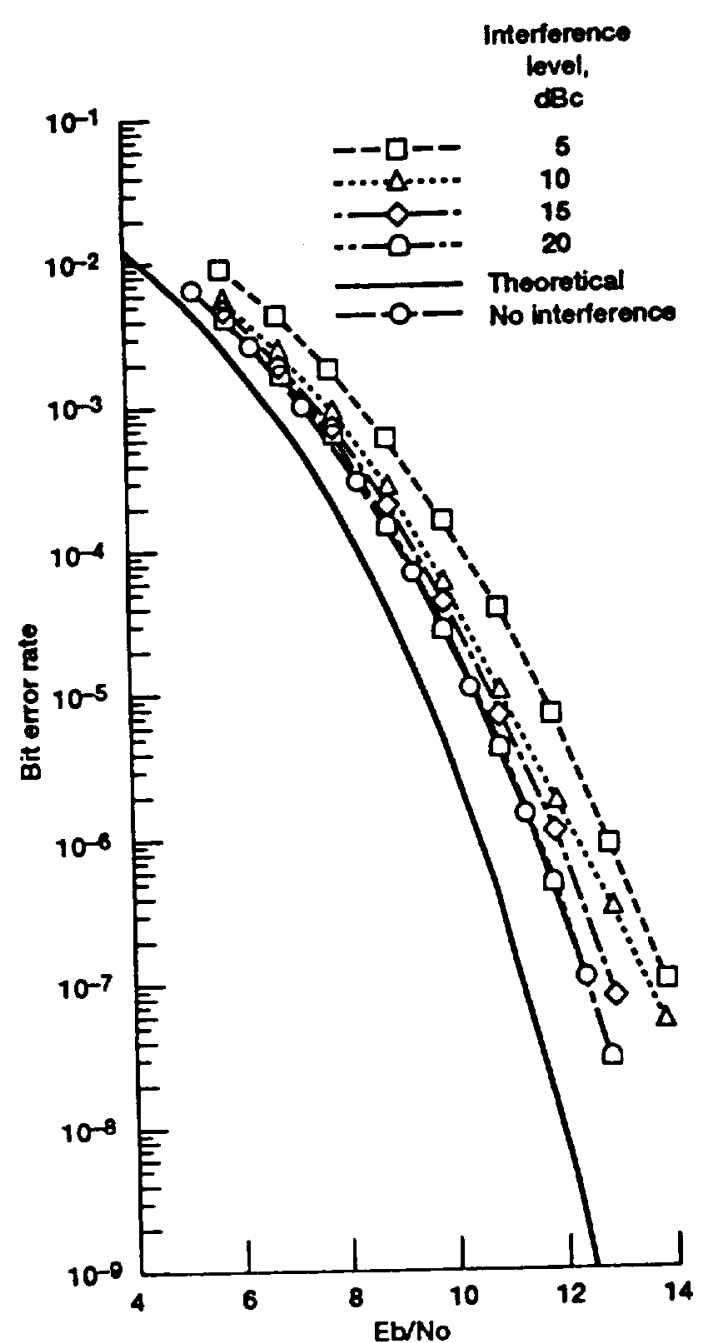

Figure 7.-BER curves for uplink modulated interference in the adjacent channel below the desired signal.

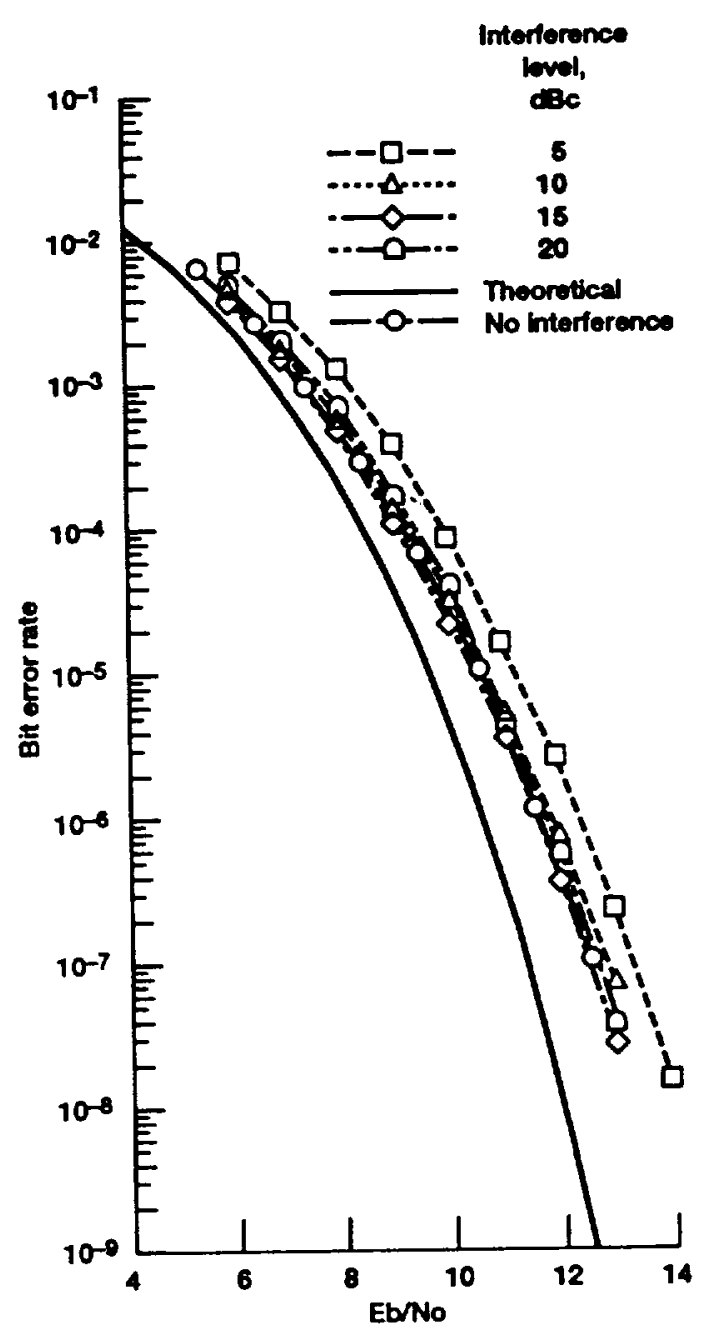

Figure 8.-BER curves for uplink modulated interference in the adjacent channel above the desired signal. 


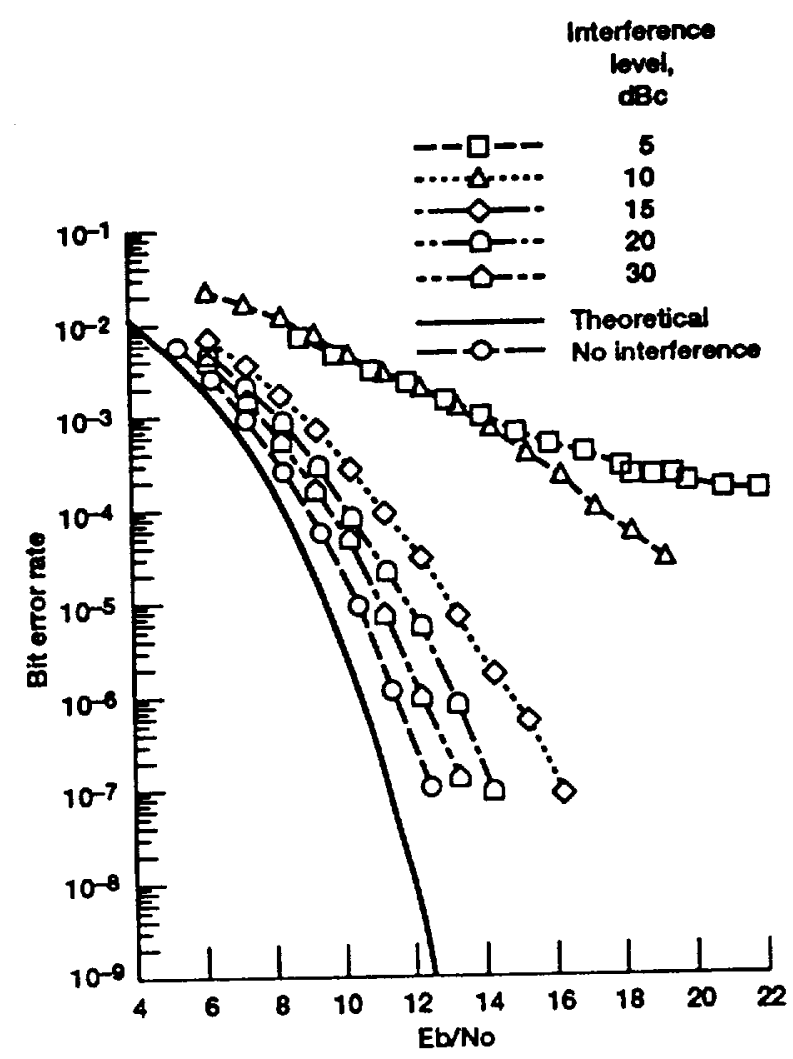

Figure 9.-BER curves for uplink modulated cochannel interference.

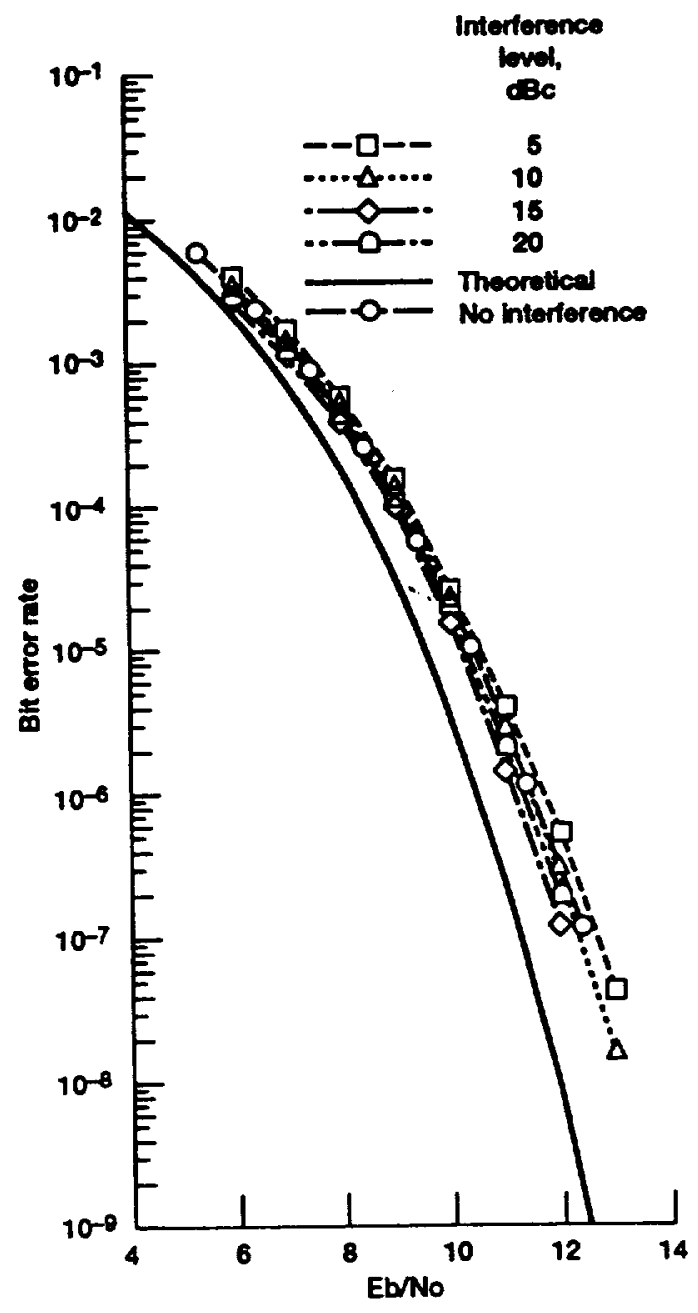

Figure 10.-BER curves for downlink modulated interference in the adjacent channel below the desired signal. 


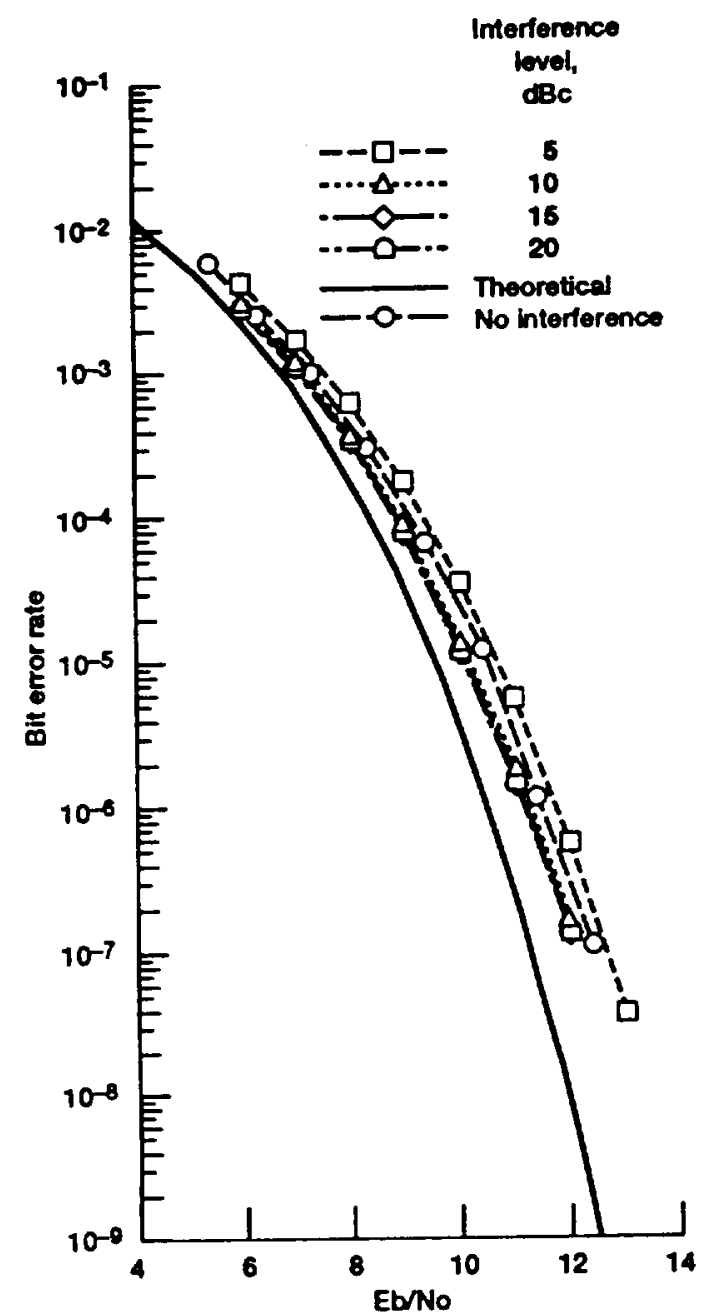

Figure 11. - BER curves for downlink modulated interference in the adjacent channel above the desired signal.

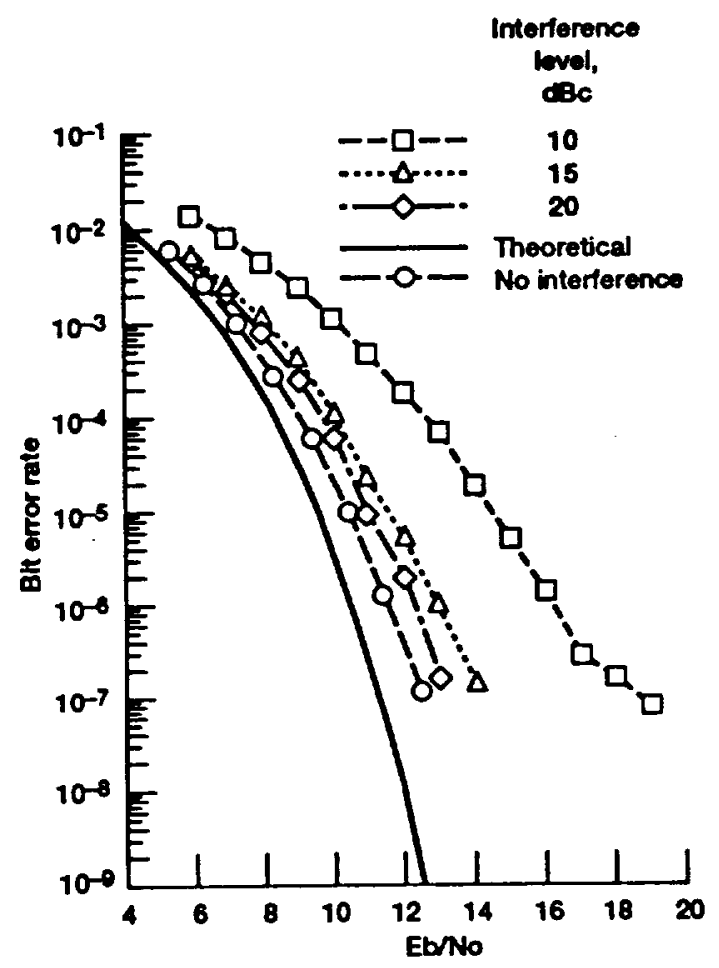

Figure 12.-BER curves for downlink modulated cochannel interference. 


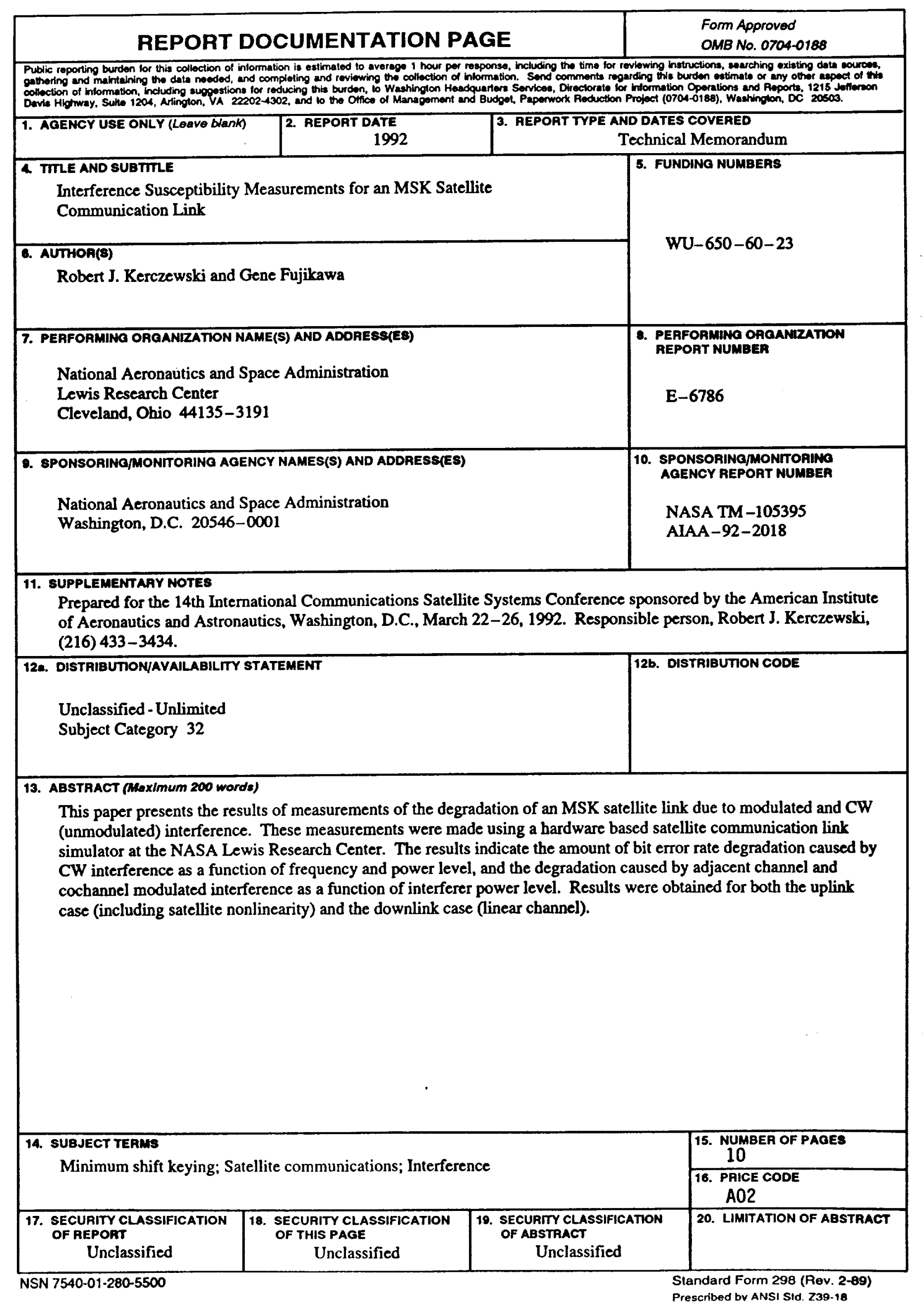

